\title{
Stability of incompressible formulations enriched with X-FEM
}

\author{
G. Legrain ${ }^{a}$ N. Moës ${ }^{a, *}$ A. Huerta ${ }^{b}$ \\ ${ }^{a}$ GeM Institute - École Centrale de Nantes / Université de Nantes / CNRS \\ 1 Rue de la Noë, 44321 Nantes, France. \\ ${ }^{\mathrm{b}}$ Laboratori de Càlcul Numèric (LaCàN) - Edifici C2, Campus Nord, Universitat \\ Politècnica de Catalunya - E-08034 Barcelona, Spain.
}

\begin{abstract}
The treatment of (near-)incompressibility is a major concern for applications involving rubber-like materials, or when important plastic flows occurs as in forming processes. The use of mixed finite element methods is known to prevent the locking of the finite element approximation in the incompressible limit. However, it also introduces a critical condition for the stability of the formulation, called the infsup or LBB condition. Recently, the finite element method has evolved with the introduction of the partition of unity. The eXtended Finite Element Method (XFEM) uses the partition of unity to remove the need to mesh physical surfaces or to remesh them as they evolve. The enrichment of the displacement field makes it possible to treat surfaces of discontinuity inside finite elements. In this paper, some strategies are proposed for the enrichment of mixed finite element approximations in the incompressible setting. The case of holes, material interfaces and cracks are considered. Numerical examples show that for well chosen enrichment strategies, the finite element convergence rate is preserved and the inf-sup condition is passed.
\end{abstract}

Key words: Mixed formulation, X-FEM, Partition of Unity, inf-sup condition, incompressibility, holes, inclusions, fracture mechanics

\footnotetext{
* Corresponding author

Email addresses: gregory.legrain@ec-nantes.fr(G. Legrain), nicolas.moes@ec-nantes.fr (N. Moës), antonio.huerta@upc.edu (A. Huerta).
}

Preprint submitted to Elsevier Science 13 September 2005 


\section{Introduction}

Displacement based finite element methods are nowadays abundantly used in engineer analysis. Indeed, they can solve a wide variety of problems, and have now been deeply mathematically investigated. However there still exists two main drawbacks for these methods. First, the treatment of incompressible or nearly incompressible problems necessitates the use of adapted formulations. If not, incompressibility constraint locks the approximation, leading for instance to non-physical displacement fields. Second, the generation and especially the update of the mesh in complex 3D settings for evolving boundaries such as cracks, material interfaces and voids still lacks robustness, and involves important human effort.

Several techniques have been developed to respond to the locking issue. For instance, the selective-reduced-integration procedures [1-3] or the Bbar approach of Hugues [4] in which the volumetric part of the strain tensor is evaluated at the center of the element. Another way to avoid locking is to enhance the strain tensor in order to enlarge the space on which the minimization is performed, and meet the divergence-free condition (enhanced assumed strain methods, see [5-9]).

Here, we will focus on two-field mixed finite element methods. The incompressibility constraint is weakened by the introduction of the pressure field. This alleviates locking at the price of additional pressure unknowns. However, mixed finite element methods are not stable in all cases, some of them showing spurious pressure oscillations if displacement and pressure spaces are not chosen carefully. To be stable, a mixed formulation must verify consistency, ellipticity and the so called inf-sup (or LBB) condition. The later is a severe condition which depends on the connection between the displacement and pressure approximation spaces. Stable mixed formulations can be obtained by stabilizing non-stable formulations with the use of parameters whose values may depend on the problem at hand. Otherwise, one has to work with approximation spaces which passe the inf-sup condition. To prove that a displacement-pressure pair satisfies the inf-sup condition is not a trivial task. However, a numerical test has been proposed by Brezzi and Fortin [10] then by Chapelle et al. [11], in order to draw a prediction on the fulfilment of the inf-sup. This test proved to be useful for the study of the stability of various mixed elements [12].

The second drawback of classical finite element methods (evolving boundaries) has been overcome by the development of alternative methods such as meshless methods in which the connectivity between the nodes is no longer obtained by the mesh, but by domains of influence which can be split by the boundaries. Moreover, the approximation basis can be enriched with functions coming from the physical knowledge of the problem. Note that in the incompressible limit, meshless methods are now known to lock $[13,14]$ as classical finite elements. Thus, some strategies have been developed to circumvent this 
issue [13,15]. Apart from mixing meshless methods and finite elements [16] another alternative to overcome the re-meshing issue in finite elements is to use the eXtended Finite Element Method (X-FEM) based on the partition of unity framework introduced by Babuška and Melenk [17]. Proper enrichment of the finite element basis makes it possible to model crack, material inclusions and holes with non-conforming meshes. The X-FEM method has been used for the simulation of a wide variety of problems such as fracture mechanics problems (2D[18-20], 3D[21-23], plates [24,25], cohesive zone modeling [26,27], dynamic fracture [28], nonlinear fracture mechanics[29-31]), holes [32,33], but also material inclusions [33,34] or multiple phase flows [35].

Here, we focus on the application of this method to mixed formulations for the treatment of holes, material inclusions and cracks in the incompressible limit. Bbar or selective-reduced formulations are not considered, because they do not seem to be generalized easily to enriched displacement fields. The main contribution of this paper is the design of enrichment strategies for the pressure and displacement fields, so that it leads to a stable formulation. The enrichment of mixed finite element approximations has already been used by Dolbow et al. [25] and Areias et al.[31] for fracture mechanics in plates and shells, and by Wagner et al. [36] for rigid particles in Stokes flow. However, the stability and the convergence of these approaches was not studied. The latest work concerning volumetric incompressibility was proposed by Dolbow and Devan [29]. In this papers, the authors focus on the application of the enhanced assumed strain method to X-FEM in large strain. This approach seems to lead to a stable low order formulation in the case of nearly incompressible nonlinear fracture mechanics. However, the stability of the method was not shown, and the influence of the near-tip enrichment was not studied. More precisely, it is not clear whether the near-tip enrichment could make this approach unstable, as the construction of an orthogonal enhanced strain field becomes difficult with non polynomial functions.

The paper is organized as follows: first, the governing equations of incompressible linear elasticity are recalled. The conditions for the stability of mixed formulations are also reviewed. Next, some strategies are proposed to keep the stability of enriched finite elements. The case of holes, material interfaces and 2D cracks are presented. Finally, in a last section the stability of these strategies is investigated.

\section{Governing Equations}

In this section, we focus on the design of stable mixed formulations for the treatment of incompressible elasticity. First, the equations governing incompressible linear elasticity are recalled. Then the inf-sup condition is presented together with a numerical test. 


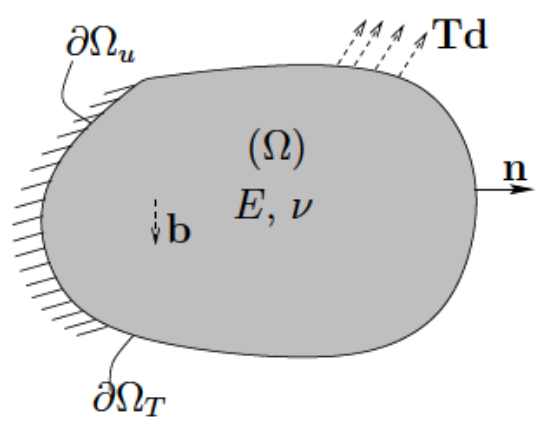

Fig. 1. The model problem.

We consider the static response of an elastic body which occupies a bounded domain $\Omega \in \mathbb{R}^{2}$ with a sufficiently smooth boundary $\partial \Omega$ which is split into two disjointed parts: $\partial \Omega_{u}$ where displacements are prescribed (Dirichlet boundary conditions) and $\partial \Omega_{T}$ where tractions are prescribed (Neumann boundary conditions). The body is initially in an undeformed, unstressed state. The governing equations are:

$$
\begin{cases}\operatorname{div} \underline{\underline{\sigma}}+\mathbf{b}=\mathbf{0} & \text { on } \Omega \\ \underline{\underline{\varepsilon}}=\frac{1}{2}\left(\nabla \mathbf{u}+\nabla \mathbf{u}^{T}\right) & \text { on } \Omega \\ \underline{\underline{\sigma}} \cdot \mathbf{n}=\mathbf{T}_{\mathbf{d}} & \text { on } \partial \Omega_{T} \\ \mathbf{u}(\mathbf{x})=\mathbf{u}_{\mathbf{d}} & \text { on } \partial \Omega_{u} \\ \underline{\underline{\sigma}}=\underline{\underline{\underline{\mathcal{C}}}}: \underline{\underline{\varepsilon}} & \text { on } \Omega\end{cases}
$$

where $\underline{\underline{\sigma}}$ is the cauchy stress tensor, $\mathbf{b}$ is the load per unit volume, $\mathbf{u}_{\mathbf{d}}$ is the prescribed displacement field, $\mathbf{T}_{\mathbf{d}}$ are the prescribed tractions, $\mathbf{n}$ is the outward unit normal to the boundary $\partial \Omega, \underline{\underline{\varepsilon}}$ is the linearized strain tensor and $\underline{\underline{\underline{\mathcal{C}}}}$ is the fourth order elasticity tensor which must be bounded, i.e.

$$
\mathcal{C}_{i j k l} \in L^{\infty}(\Omega) \quad i, j, k, l=1,2,3
$$

In the case of a linear isotropic elastic material, the constitutive equation can be written as:

$$
\underline{\underline{\sigma}}=\kappa \underline{\underline{\varepsilon}}_{V}(\mathbf{u}) \underline{\underline{I}}+2 \mu \underline{\underline{\varepsilon}}^{D}(\mathbf{u}) \quad \text { in } \Omega
$$

where $\varepsilon_{V}$ is the volumetric strain $\left(\varepsilon_{V}(\mathbf{u})=\operatorname{div} \mathbf{u}\right), \kappa$ is the bulk modulus,

$$
\kappa=\frac{E}{3(1-2 \nu)}
$$


and $\underline{\underline{\varepsilon}}^{D}$ is the deviatoric strain operator:

$$
\underline{\underline{\varepsilon}}^{D}=\underline{\underline{\varepsilon}}-\frac{\varepsilon_{V}}{3} \underline{\underline{I}}
$$

When the material tends to incompressibility, the bulk modulus tends to infinity. This means that $\varepsilon_{V}$ must tend to zero in order to meet condition (2) (the displacement field must be divergence free in the incompressible limit).

$$
\varepsilon_{V}=\operatorname{div}(\mathbf{u}) \longrightarrow 0 \text { as } \nu \rightarrow 0.5
$$

The strong form (1) is equivalent to the stationarity of a displacement potential $\Pi$ :

$$
\Pi(\mathbf{u})=\frac{1}{2} \int_{\Omega} \underline{\underline{\varepsilon}}(\mathbf{u}): \underline{\underline{\underline{\underline{\mathcal{C}}}}}: \underline{\underline{\varepsilon}}(\mathbf{u}) d \Omega-\int_{\Omega} \mathbf{u} \cdot \mathbf{b} d \Omega-\int_{\partial \Omega_{t}} \mathbf{u} \cdot \mathbf{T}_{\mathbf{d}} d \Gamma
$$

In order to model incompressible or almost incompressible problems, a two field principle is considered by introducing a second variable (the hydrostatic pressure $p$ ) in the potential (7):

$$
p=-\kappa \varepsilon_{V}(\mathbf{u})=-\frac{1}{3} \operatorname{Tr}(\underline{\underline{\sigma}})
$$

When $\kappa$ increases, the volumetric strain $\varepsilon_{V}$ decreases and becomes very small. For total incompressibility, the bulk modulus is infinite, the volumetric strain is zero, and the pressure remains finite (of the order of the applied boundary tractions). The stress tensor is then expressed as:

$$
\underline{\underline{\sigma}}=-p \underline{\underline{I}}+2 \mu \underline{\underline{\varepsilon}}^{D} \quad \text { in } \Omega
$$

The solution of the governing differential equations (1) now involves two variables: the displacement field and the pressure field. Writing the two fields variational principle, the total potential for the $u-p$ formulation is expressed as:

$$
\begin{aligned}
\chi(\mathbf{u}, p)=\frac{1}{2} \int_{\Omega} \underline{\underline{\varepsilon}}^{D}(\mathbf{u}): \underline{\underline{\underline{\mathcal{C}}}: \underline{\underline{\varepsilon}}^{D}(\mathbf{u}) d \Omega} & -\int_{\Omega} \mathbf{u} \cdot \mathbf{b} d \Omega-\int_{\partial \Omega_{t}} \mathbf{u} \cdot \mathbf{T}_{\mathbf{d}} d \Gamma \\
& -\frac{1}{2} \int_{\Omega} \frac{p^{2}}{\kappa} d \Omega-\int_{\Omega} p \varepsilon_{V}(\mathbf{u}) d \Omega
\end{aligned}
$$

Invoking the stationarity of $\chi(\mathbf{u}, p)$ with respect to the two independent variables $\mathbf{u}$ and $p$, we obtain:

$$
\begin{aligned}
& \int_{\Omega} \delta \underline{\underline{\varepsilon}}^{D}: \underline{\underline{\underline{\mathcal{C}}}}: \underline{\underline{\varepsilon}}^{D} d \Omega-\int_{\Omega} p \delta \varepsilon_{V} d \Omega=R(\delta \mathbf{v}) \\
& -\int_{\Omega}\left(\frac{p}{\kappa}+\varepsilon_{V}\right) \delta p d \Omega=0
\end{aligned}
$$

where $R(\delta \mathbf{v})$ represents the virtual work of the external loads. In the case of 


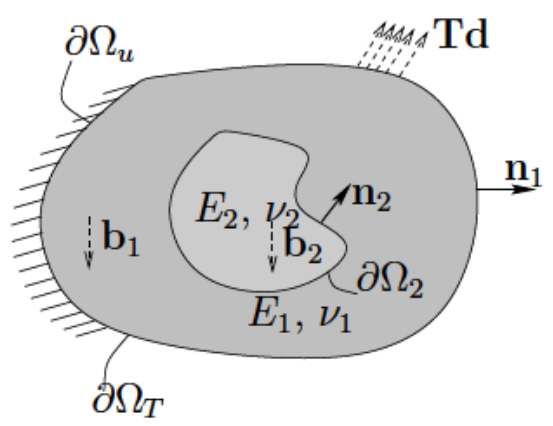

Fig. 2. The model problem with material interfaces.

two perfectly bounded materials, each phase of the body must satisfy (1) in addition to continuity conditions:

$$
\begin{aligned}
& \llbracket \mathbf{u} \rrbracket=\mathbf{0} \quad \text { on } \partial \Omega_{2}
\end{aligned}
$$

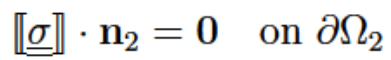

where the jump operator $\llbracket \cdot \rrbracket$ is defined along the interface, $\partial \Omega_{2}$, as $\llbracket \mathbf{v} \rrbracket=$ $\mathbf{v}_{1}-\mathbf{v}_{2}$. Remark that (14) does not implies that the pressure, nor the strain field, are continuous across the interface.

\subsection{Stability of mixed formulations}

\subsubsection{Analytical condition for stability - the inf-sup condition}

The discretization of equation (11) leads to a finite element system in the form (see section 3):

$$
\left(\begin{array}{cc}
\mathbf{K}_{\mathrm{uu}} & \mathrm{K}_{\mathrm{up}} \\
\mathrm{K}_{\mathrm{up}}{ }^{T} & \frac{1}{\kappa} \mathbf{K}_{\mathrm{pp}}
\end{array}\right)\left(\begin{array}{l}
\mathbf{u} \\
\mathbf{p}
\end{array}\right)=\left(\begin{array}{l}
\mathrm{f} \\
\mathbf{0}
\end{array}\right)
$$

In order to be regular in the full range of $\kappa$, the kernel of the $\mathbf{K}_{\mathrm{up}}$ matrix must be zero (as well of course as appropriate removal of the rigid modes) More precisely, the displacement and pressure interpolations must satisfy the Ladyzhenskaya - Babuška - Brezzi compatibility condition [10], also known as the LBB (or inf-sup) condition. This condition states that the displacement and pressure spaces cannot be chosen separately. To ensure solvability, a necessary but not sufficient condition for the uniqueness of $\mathbf{u}$ and $p$ is that:

$$
\operatorname{dim} \mathcal{Q}^{h} \leqslant \operatorname{dim} \mathcal{V}^{h}
$$

where $\mathcal{Q}^{h}$ and $\mathcal{V}^{h}$ are respectively the pressure and displacement finite element spaces. The sufficient condition linking these spaces (inf-sup or LBB condition) 
expresses as:

The existence of a stable finite element approximate solution $\left(\mathbf{u}^{h}, p^{h}\right)$ depends on choosing a pair of spaces $\mathcal{V}^{h}$ and $\mathcal{Q}^{h}$ such that the following condition holds:

$$
\inf _{q^{h} \in \mathcal{Q}^{h}} \sup _{v^{h} \in \mathcal{V}^{h}} \frac{\int_{\Omega} q^{h} \operatorname{div} \mathbf{v}^{h} d \Omega}{\left\|\mathbf{v}^{h}\right\|_{1}\left\|q^{h}\right\|_{0}} \geqslant \beta>0
$$

where $\|\cdot\|_{1}$ and $\|\cdot\|_{0}$ indicates $H^{1}$ and $L^{2}$ norms respectively and $\beta$ is independent of the mesh size $h$. If the inf-sup compatibility condition is satisfied, then there exists a unique $\mathbf{u}^{h} \in \mathcal{V}^{h}$ and a $p^{h} \in \mathcal{Q}^{h}$ (determined up to an arbitrary constant in the case of purely Dirichlet boundary conditions).

\subsubsection{Numerical assessment of the inf-sup condition}

As seen before, the prediction of the stability of a mixed formulation involves the fulfillment of the inf-sup criterion. This criterion is however impossible to prove for practical situations. This is why the numerical evaluation of the inf-sup condition has received considerable attention $[11,10]$. This numerical evaluation, although not equivalent to the analytical inf-sup, gives indications on whether (17) is fulfilled or not for a given set of finite element discretizations. The numerical inf-sup test is based on the following theorem.

Proposition $\mathbf{1}$ Let $\mathbf{M}_{\mathbf{u u}}$ and $\mathbf{M}_{\mathbf{p p}}$ be the mass matrices associated to the scalar products of $\mathcal{V}^{h}$ and $\mathcal{Q}^{h}$ respectively and let $\mu_{\text {min }}$ be the smallest non zero eigenvalue defined by the following eigenproblem:

$$
\mathbf{K}_{\text {up }}^{T} \mathbf{M}_{\mathbf{u u}}^{-1} \mathbf{K}_{\mathbf{u p}} \mathbf{q}=\mu^{2} \mathbf{M}_{\mathbf{p p}} \mathbf{q}
$$

then the value of $\beta$ is simply $\mu_{\min }$.

The proof can be found in [37] or [10]. The numerical test proposed in [11] consists in testing a particular formulation by calculating $\beta$ using meshes of increasing refinement. On the basis of three or four results it can be predicted whether the inf-sup value is probably bounded from underneath or, on the contrary, goes down to zero when the mesh is refined. The reliability of this test is demonstrated on several examples of elements for incompressible elasticity problems in [11]. In the following section this test is used to check the behavior of proposed enrichment strategies. However, we follow [11] and use only $\mathbf{S}_{\mathbf{u u}}=$ $\int_{\Omega} \underline{\underline{\nabla}} \mathbf{u}: \underline{\underline{\nabla}} \mathbf{u} d \Omega$ instead of $\mathbf{M}_{\mathbf{u u}}$ in (18). In order to perform the numerical infsup test, a sequence of successive refined meshes is considered. The objective is to monitor the inf-sup values, $\beta$, when $h$ decreases. If a steady decrease in $\log (\beta)$ is observed when $\mathrm{h}$ goes to zero, the element is predicted to violate the inf-sup condition and said to fail the numerical test. But, if the $\log (\beta)$ value is stable as the number of elements increases, the test is numerically passed. 


\section{X-FEM Discretization}

\subsection{Displacement field}

With classical finite elements, the approximation of a vector field $\mathbf{u}$ on an element $\Omega_{e}$ is written as:

$$
\left.\mathbf{u}(\mathbf{x})\right|_{\Omega_{e}}=\sum_{\alpha=1}^{n_{u}} u^{\alpha} \mathbf{N}_{\mathbf{u}}^{\alpha}(\mathbf{x})
$$

where $n_{u}$ is the number of coefficients describing the approximation of the displacement over the element, $u^{\alpha}$ is the $\alpha^{\text {th }}$ coefficient of this approximation and $\mathbf{N}_{\mathbf{u}}^{\alpha}$ is the vectorial shape function associated to the coefficient $u^{\alpha}$. Within the partition of unity, the approximation is enriched as:

$$
\left.\mathbf{u}(\mathbf{x})\right|_{\Omega_{e}}=\sum_{\alpha=1}^{n_{u}} \mathbf{N}_{\mathbf{u}}^{\alpha}\left(u^{\alpha}+\sum_{\beta=1}^{n_{e n r}} a_{\beta}^{\alpha} \phi_{\beta}^{u}(\mathbf{x})\right)
$$

where $n_{e n r}$ is the number of enrichment modes, $a_{\beta}^{\alpha}$ is the additional dof associated to dof $\alpha$ and $\phi_{\beta}^{u}$ stands for the $\beta^{\text {th }}$ scalar enrichment function. The number and the expression of the enrichment functions vary with the problem to model. The expression of this enrichment function will be recalled for holes, inclusions and fracture mechanics in the next sections.

\subsection{Pressure field}

Using the same scheme, the pressure approximation is written as:

$$
\left.p(\mathbf{x})\right|_{\Omega_{e}}=\sum_{\alpha=1}^{n_{p}} N_{p}^{\alpha}\left(p^{\alpha}+a^{\alpha} \phi^{p}(\mathbf{x})\right)
$$

where $n_{p}$ is the number of coefficients describing the approximation over the element, $p^{\alpha}$ is the $\alpha^{\text {th }}$ coefficient of this approximation and $N_{p}^{\alpha}$ is the scalar shape function associated to the coefficient $p^{\alpha}, a^{\alpha}$ is the additional dof associated to dof $\alpha$ and $\phi^{p}$ stands for the scalar pressure enrichment function.

The key issue is the combined choice of enrichment functions $\phi^{u}$ and $\phi^{p}$ such that the whole enriched approximation (displacement and pressure) passes the inf-sup condition. 


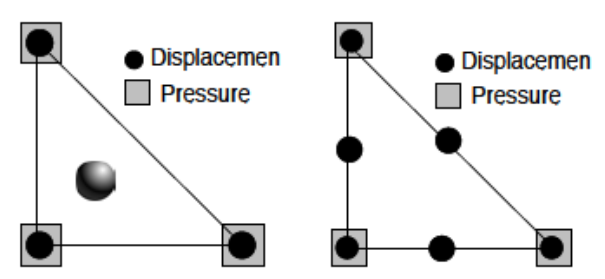

Fig. 3. The Mini element (left) and T6T3 element (right).

\subsection{Stable mixed finite elements}

In this work, we shall enrich two stable mixed finite elements: the Mini element (see Figure 3) introduced by Arnold, Brezzi and Fortin [38] considering linear displacement field plus a bubble function and linear pressure field $\left(P_{1}^{+}, P_{1}\right.$ element). Second, the T6T3 $\left(P_{2}, P_{1}\right.$ element) (see Figure 3$)$ which is a robust and classical mixed element.

\section{Stability of mixed formulations enriched with X-FEM}

\subsection{The case of Holes}

The strategy for treating holes within the X-FEM consists in an integration of the weak form only in the non-void parts of the elements (See [32,33]). Moreover, the nodes for which the support is completely in the void are eliminated. In the incompressible setting, it is natural to follow the same strategy. No enrichment will be used, and the weak form will be integrated in the body only.

The stability of this strategy is evaluated by considering convergence studies under total incompressibility, and numerical evaluation of the inf-sup. The strategy will be considered as stable if the finite element convergence rate is shown to be preserved, and if the numerical inf-sup test is passed.

\subsubsection{Convergence study}

Consider an infinite plate with a traction-free circular hole under uniaxial tension. The exact solution of this problem is given in [33]. We consider a square domain of edge length 2 with a circular hole of radius $a=0.4$ at its center. The exact tractions are imposed on the boundaries of the domain and rigid body modes are prevented. The Poisson's ratio is set to 0.5 (total incompressibility), and the Young's modulus to 1.0. In addition, plane strain conditions are assumed. The sequence of meshes that we consider for the convergence study are unstructured and do not match the hole boundary. The 
displacement error is measured using the energy norm error defined Eq. (22), and the error on the pressure is computed using the $L^{2}$ norm (23).

$$
\begin{gathered}
\varepsilon_{u}=\frac{\left(\int_{\Omega}\left(\underline{\underline{\varepsilon}}\left(u^{h}\right)-\underline{\underline{\varepsilon}}(u)\right): \underline{\left.\underline{\underline{\underline{\mathcal{C}}}}:\left(\underline{\underline{\varepsilon}}\left(u^{h}\right)-\underline{\underline{\varepsilon}}(u)\right) d \Omega\right)^{1 / 2}}\right.}{\left(\int_{\Omega} \underline{\underline{\varepsilon}}(u): \underline{\underline{\underline{\underline{\mathcal{C}}}}: \underline{\underline{\varepsilon}}(u) d \Omega)^{1 / 2}}\right.} \\
\varepsilon_{p}=\frac{\left(\int_{\Omega}\left(p^{h}-p\right)^{2} d \Omega\right)^{1 / 2}}{\left(\int_{\Omega} p^{2} d \Omega\right)^{1 / 2}}
\end{gathered}
$$

The convergence study is performed using both Mini and T6T3 elements, and the results are presented in Figure 4 for both pressure and displacement. As
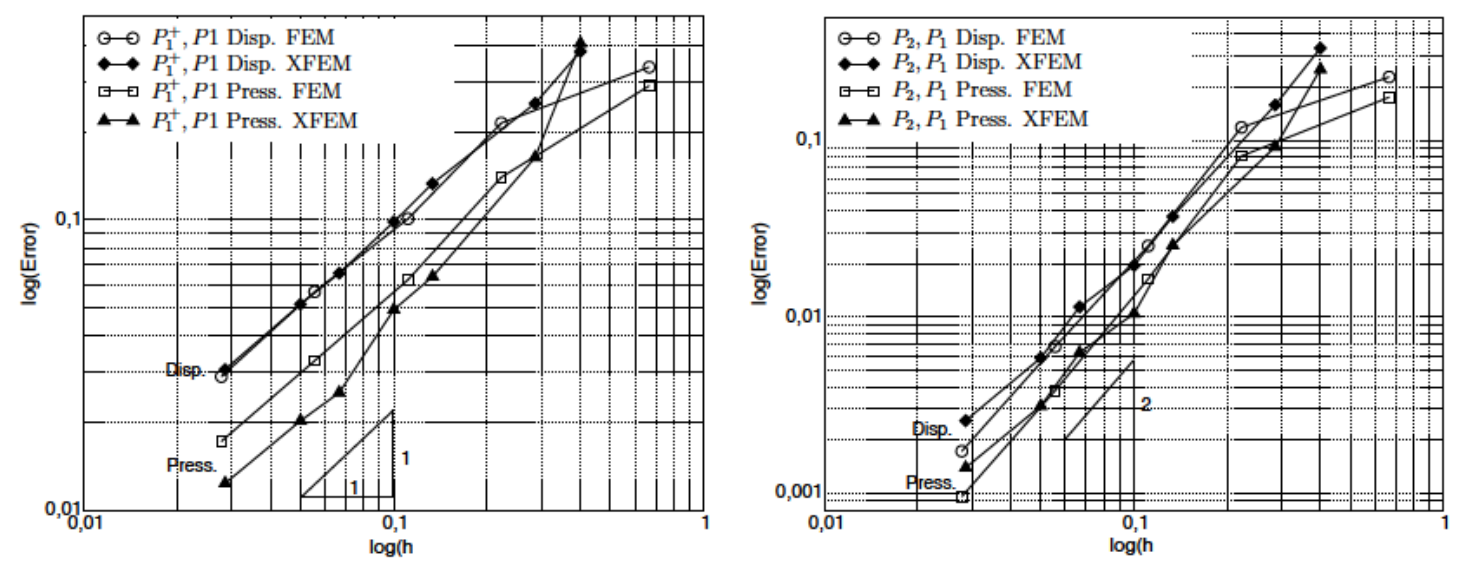

Fig. 4. Convergence study, incompressible plate with a hole. Left: Mini, right: T6T3.

seen on Figure 4, the convergence rate of both displacement and pressure are similar to the theoretical one. This demonstrates that the X-FEM strategy for the treatment of holes preserves the convergence properties of Mini and T6T3 elements.

\subsubsection{Numerical Inf-sup test}

Convergence study was a first step to assess the behavior of the X-FEM strategy for holes. The evaluation of the fulfillment of the inf-sup condition is another approach for the validation. We follow the work of Chapelle and Bathe [11] described in section 2.2.2, and consider the problem presented in Figure 5(a). It is composed of a square of length 2, with a hole of radius 0.4 at its center. The square has its bottom and left edges blocked, and a pressure is applied on the upper edge. The inf-sup value is approximated using gradually refined structured triangular meshes. Mini and T6T3 formulations are considered herein. The evolution of the numerical inf-sup value is plotted in figure $5(\mathrm{~b})$ with respect to the element size. As seen on this figure, the numerical inf-sup value is stable for Mini and T6T3 formulations. 


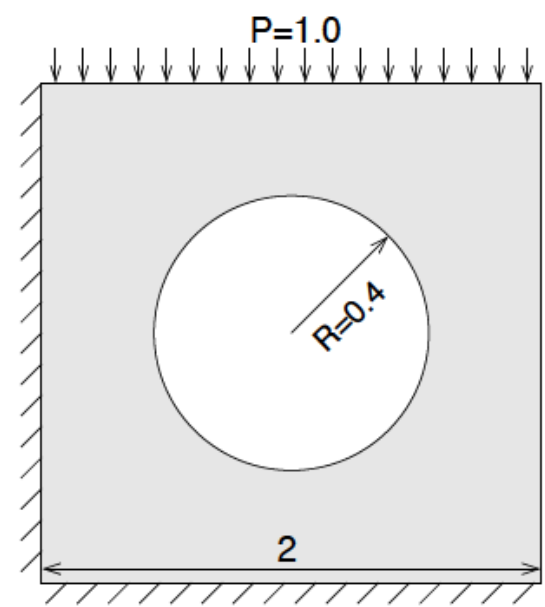

(a)

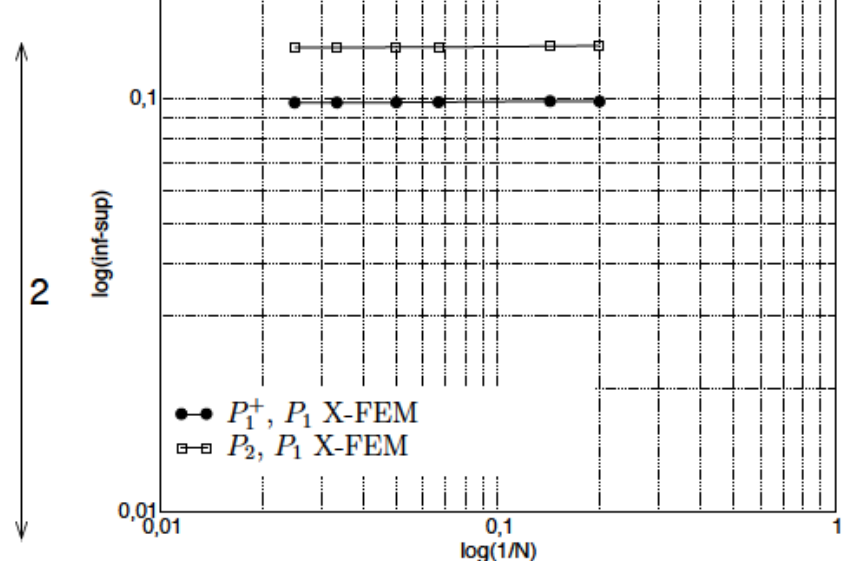

(b)

Fig. 5. Inf-sup evaluation for holes: (a) model problem, (b) Evolution of the numerical inf-sup.

\subsection{Material inclusions}

The treatment of material inclusion in a compressible media has been treated in various contributions (see for example $[33,34]$ ). In this paper, we focus on the enrichment strategy proposed by Moës et al. in [34] because this enrichment function preserves the finite element convergence rate (observed numerically), this "ridge" function is expressed as:

$$
\phi^{u}(\mathrm{x})=\sum_{\alpha}\left|\xi^{\alpha}\right| N^{\alpha}(\mathrm{x})-\left|\sum_{\alpha} \xi^{\alpha} N^{\alpha}(\mathrm{x})\right|
$$

where $\xi_{i}$ is the signed-distance function to the interface evaluated at the vertex of node $i$ (see Figure 6 for the example of a plate with a circular inclusion). Two types of enrichment are considered for the pressure:

- A discontinuous enrichment (Heaviside)

- A ridge function enrichment (by analogy with the strategy of enrichment of the displacement field)

The stability of these enriched elements are compared to the stability of the initial ones. The finite element computation of incompressible two phase media will be handled considering Mini and T6T3 elements. The X-FEM strategies considered are presented in Table 1.

The comparison of the strategies presented in Table 1 is performed as in the previous section: first, some convergence studies are carried out. Second, some numerical evaluation of the inf-sup value in order to complete the conclusions of the convergence studies. 


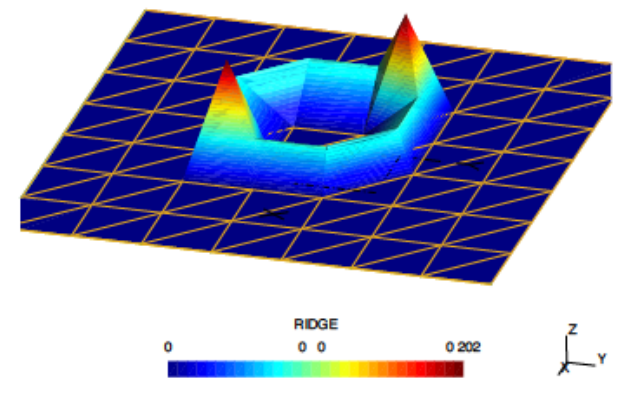

Fig. 6. Ridge function for a square plate with a circular inclusion.

\begin{tabular}{|c|c|c|c|c|}
\hline Displacement & Pressure & Formulation $\mathrm{N}^{\circ}$ & $\begin{array}{l}\text { Convergence rate } \\
\text { (Disp/Pressure) }\end{array}$ & Inf-sup test \\
\hline \multicolumn{5}{|c|}{ FEM-T6T3 } \\
\hline$P_{2}$ & $P_{1}$ & 1 & $\sim 1.5^{a} / 1.5$ & PASS \\
\hline \multicolumn{5}{|c|}{ FEM-Mini } \\
\hline$P_{1}^{+}$ & $P_{1}$ & 2 & $\sim 1 / 1$ & PASS \\
\hline \multicolumn{5}{|c|}{$X-F E M-M i n i$} \\
\hline$P_{1}^{+} \oplus P_{1}^{+} * R$ & $P_{1} \oplus P_{1} * R$ & 3 & $\sim 1 / 1$ & PASS \\
\hline$P_{1}^{+} \oplus P_{1} * R$ & $P_{1} \oplus P_{1} * R$ & 4 & $\sim 1 / 1$ & PASS \\
\hline$P_{1}^{+} \oplus P_{1}^{+} * R$ & $P_{1} \oplus P_{1} * H$ & 5 & $\sim 1 /-$ & FAIL \\
\hline$P_{1}^{+} \oplus P_{1} * R$ & $P_{1} \oplus P_{1} * H$ & 6 & $\sim 1 /-$ & FAIL \\
\hline \multicolumn{5}{|c|}{$X-F E M-T 6 T 3$} \\
\hline$P_{2} \oplus P_{2} * R$ & $P_{1} \oplus P_{1} * R$ & 7 & $\sim 1.5^{a} / 1.5$ & PASS \\
\hline$P_{2} \oplus P_{1} * R$ & $P_{1} \oplus P_{1} * R$ & 8 & $\sim 1.5^{a} / 1.5$ & PASS \\
\hline$P_{2} \oplus P_{2} * R$ & $P_{1} \oplus P_{1} * H$ & 9 & $\sim 1.5^{a} /-$ & FAIL \\
\hline$P_{2} \oplus P_{1} * R$ & $P_{1} \oplus P_{1} * H$ & 10 & $\sim 1.5^{a} /-$ & FAIL \\
\hline
\end{tabular}

$a$ Non isoparametric elements

Table 1

Approximation spaces ( $R$ : ridge enrichment, $H$ : Heaviside enrichment).

\subsubsection{Convergence studies}

Consider the example presented in Figure 7. It is composed of two perfectly bounded incompressible materials with shear modulus $\mu_{1}$ and $\mu_{2}$. The first material is held fixed at radius $r=a$ and the interface between the two materials 
is located at radius $r=b$. The structure is subjected to body forces $\mathbf{b}_{1}$ and $\mathbf{b}_{2}$ in $\Omega_{1}$ and $\Omega_{2}$ respectively, and tractions are enforced at radius $r=c$. The

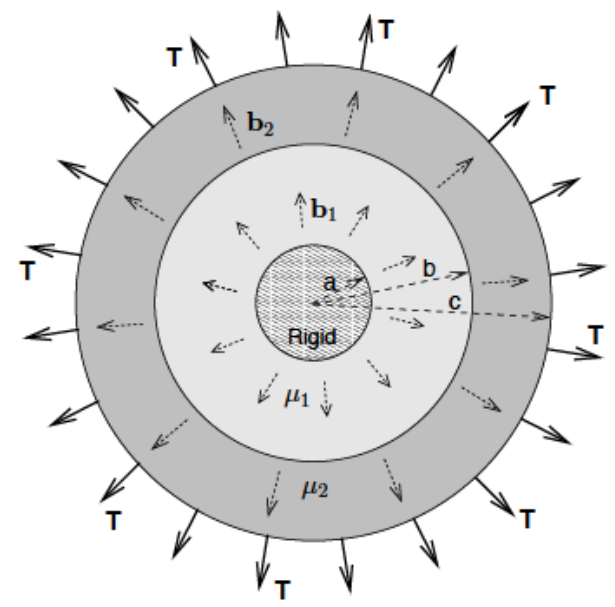

Fig. 7. Shear based example.

analytical pressure is depicted in Figure 8, and detailed in appendix A. The

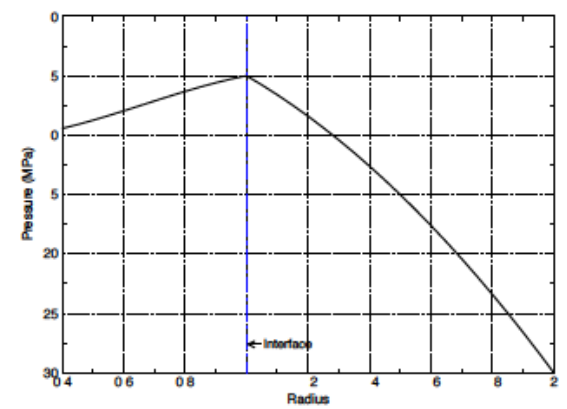

Fig. 8. Analytical pressure.

results of the convergence study are presented in Figure 9. The energy norm error on the displacement exhibits a rate of convergence in $O(h)$ independently of the enrichment strategy. This means that concerning the displacement, the theoretical rate of convergence is preserved with all the strategies. Now, consider the convergence of the pressure field which is a strong indicator on the performance of a mixed formulation. Figure 9 shows that formulations 2 and 3 give the theoretical convergence rates. Concerning formulation 5 , the convergence rate is degraded when the mesh is refined. In fact, this comes from very localized spurious pressure modes which degrades the error. This phenomenon is caused by the diminution of the so called "constraint ratio" $\gamma$ between the number of displacement dofs $n_{u}$ and the number of pressure dofs $n_{p}$ when the interface comes near the element edges. This is illustrated in Figure 10 where $\gamma$ passes from 6.0 to 4.0 if the pressure is enriched with the Heaviside function. On the contrary, in the case of the ridge enrichment, the ratio remains stable (see Figure 11).

In Figure 12, the case where only the linear part of the approximation space is enriched is considered. As seen on this figure, the rate of convergence is 


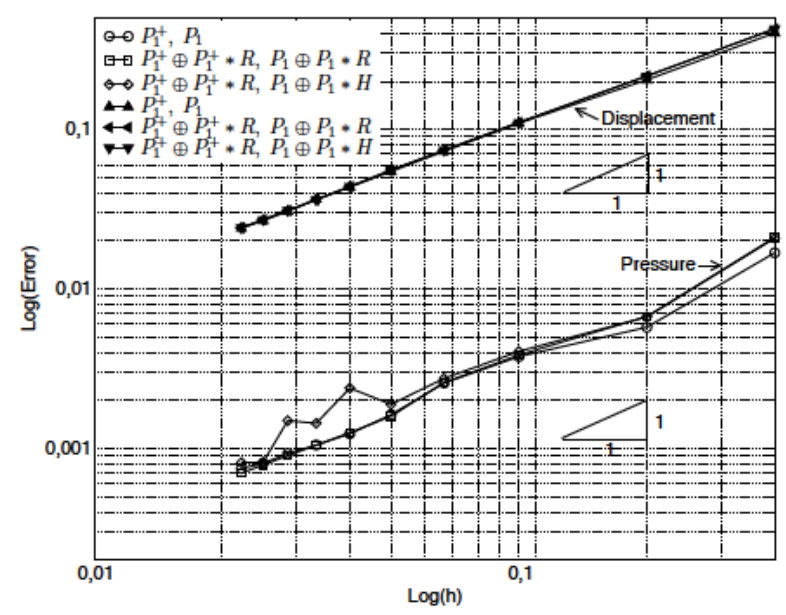

Fig. 9. Rate of convergence for the problem Fig.7.

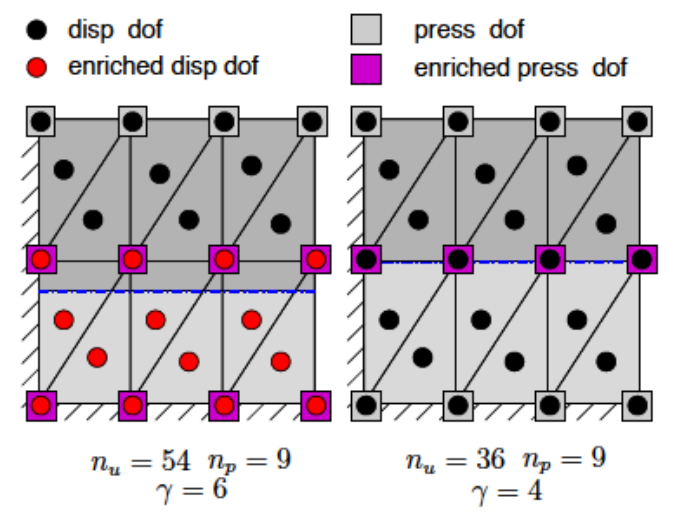

Fig. 10. Illustration of the drop in the constraint ration $(\gamma)$ with the Heaviside enrichment when the mesh matches the interface (right) or not (left).

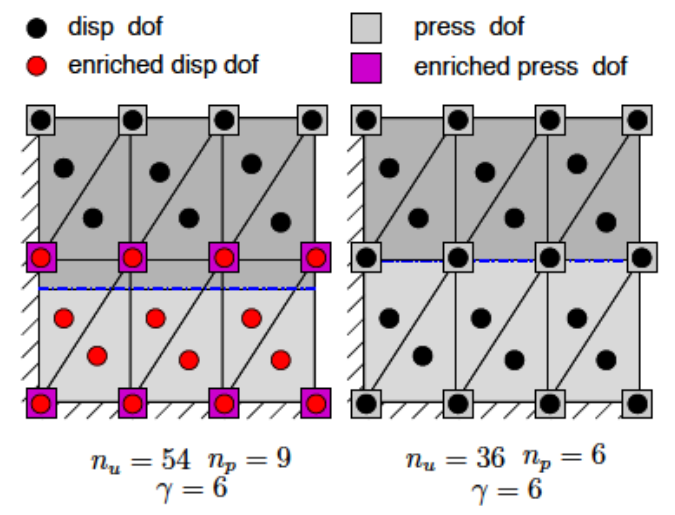

Fig. 11. Illustration of the stability of the constraint ration $(\gamma)$ with the Ridge enrichment when the mesh matches the interface (right) or not (left).

degraded for the Heaviside-based enrichment when the mesh is refined. However, with ridge-based enrichment, it remains similar to the optimal convergence rate for both pressure and displacement. Finally, the case of quadratic T6T3 based elements is considered in Figure 13: the rate of convergence is in $O\left(h^{3 / 2}\right)$ for the pressure and slightly slower for the displacement (even with 


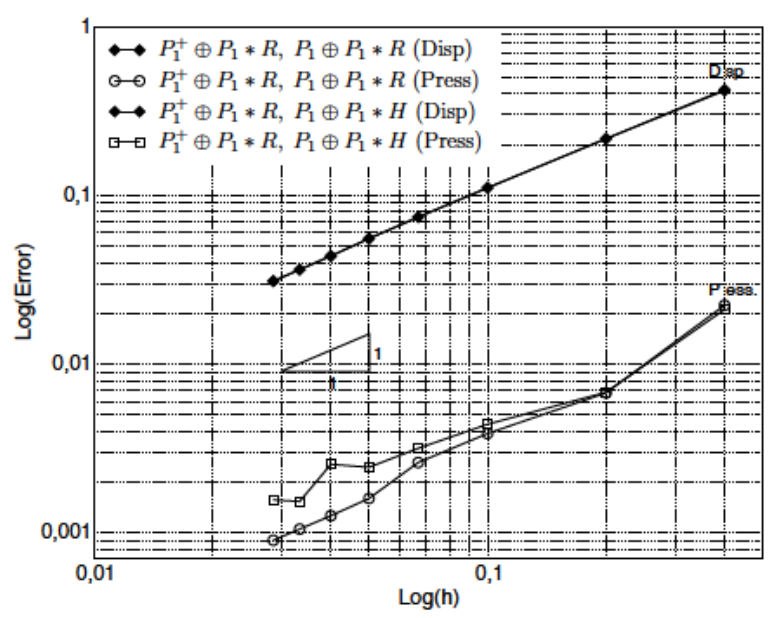

Fig. 12. Rate of convergence for the problem Fig.7, linear part enriched (Mini based element).

meshed domains), although it should be in $O\left(h^{2}\right)$ for both of them. We believe that it comes from the fact that the elements used here are not isoparametric elements (straight edge triangles). Thus, the error in the representation of curved domains degrades the rate of convergence to $O\left(h^{3 / 2}\right)$. The conclusions remain similar when only the linear part of the displacement is enriched (see figure 14). As seen in these first numerical examples, the enrichment of both

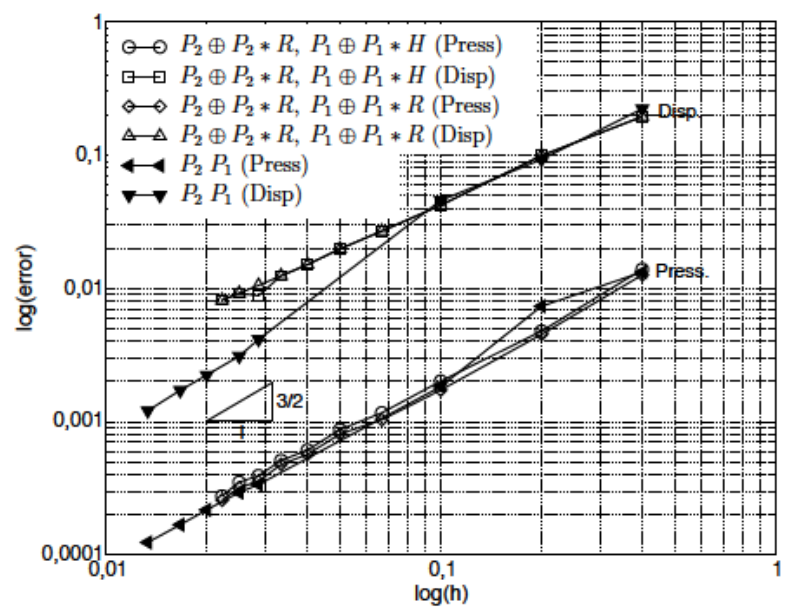

Fig. 13. Rate of convergence for the problem Fig.7, quadratic interpolation.

pressure and displacement with the ridge function gives the theoretical convergence rates for the Mini element. However, the example proposed here does not allow the full study of T6T3 based elements (even the displacement-based $\mathrm{P}_{2}$ element converge in $O\left(h^{3 / 2}\right)$ in the compressible case). Finally, we have seen that not enriching the bubble function gives similar results with lesser degrees of freedom. As a conclusion, it seems that the Mini element enriched with the ridge gives good results for total incompressibility (and thus for almost incompressible materials). 


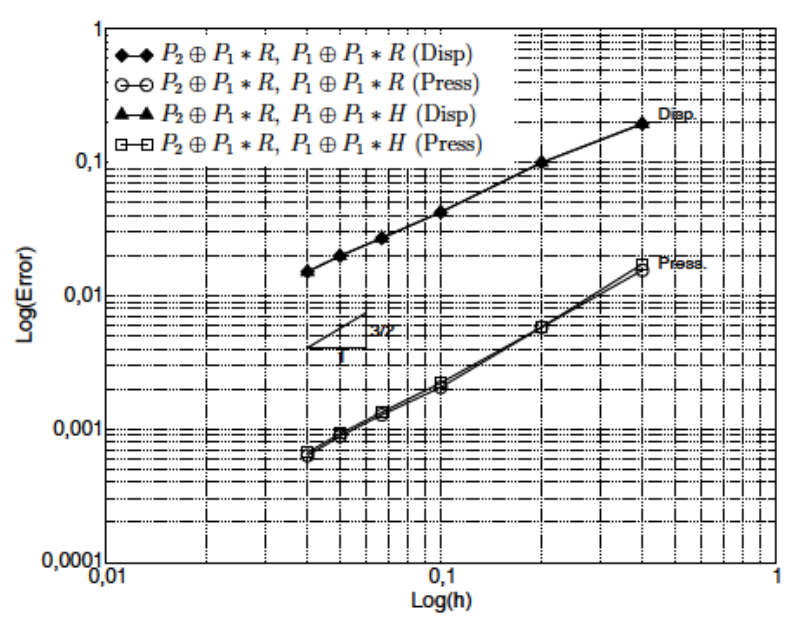

Fig. 14. Rate of convergence for the problem Fig.7, linear part enriched (T6T3 based element).

\subsubsection{Convergence studies (straight interface)}

In order to evaluate the formulations based on T6T3 elements, we have built another numerical example composed of a square of length 2 (see Figure 15) which is composed of two materials 1 and 2 of shear modulus respectively $\mu_{1}$ and $\mu_{2}$ (the case of total incompressibility is considered). The body is subjected to surface tractions $\mathbf{T}$ on its boundary, and body forces respectively $\mathbf{b}_{1}$ and $\mathbf{b}_{2}$ in 1 and 2 . The stress, strain and pressure fields are given in appendix $B$, none of them being in the finite element approximation space. Finally, we set $\mu_{1}=1 / 3$ and $\mu_{2}=10 / 3$. A convergence study is performed, and the results

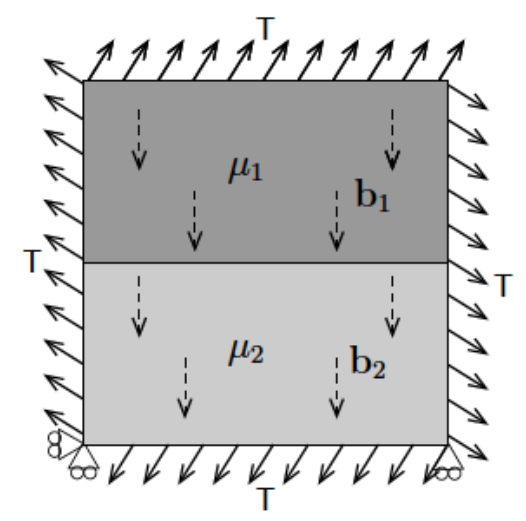

Fig. 15. Bimaterial problem with straight interface.

are given in Figure 16. We can see that T6T3 and Mini based strategies have the same rate of convergence than finite elements $(O(h)$ for the Mini and $O\left(h^{2}\right)$ for the T6T3). This shows that ridge-based enrichment seems to have a stable behaviour in the incompressible limit, and that the $O\left(h^{3 / 2}\right)$ convergence rate for T6T3 elements in the last example was caused by non-isoparametric elements. 

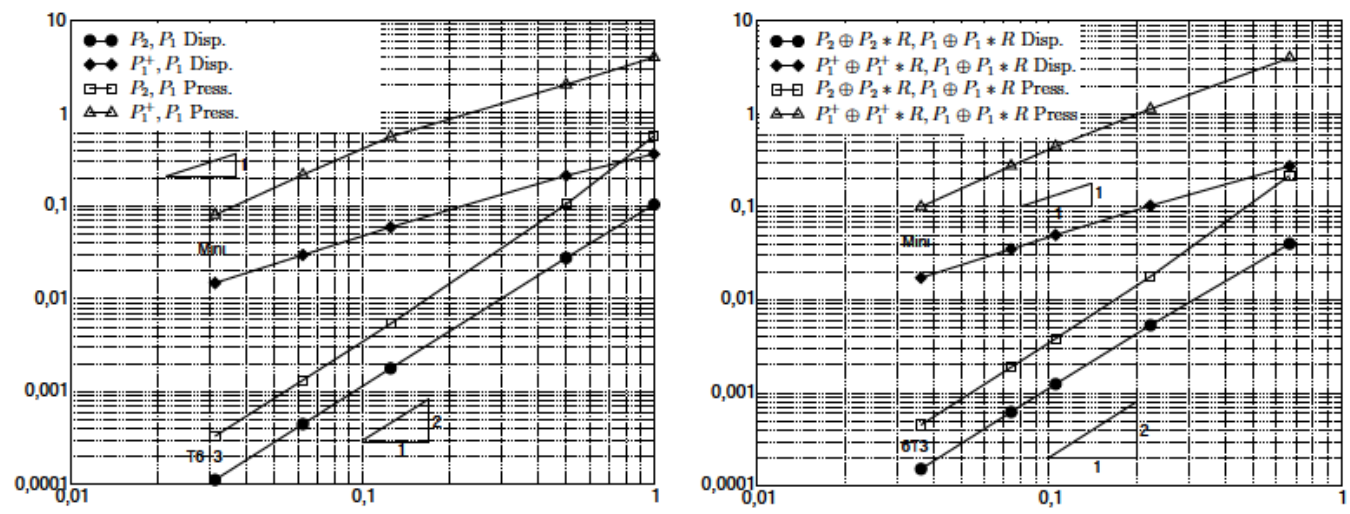

Fig. 16. Convergence study, straight interface: left FEM, right X-FEM.

\subsubsection{Numerical Inf-sup test}

To study the fulfilment of the inf-sup condition, we consider the problem presented in Figure 17, which has been adapted from [11]. It is composed of a square with two different materials subjected to a bending-type solicitation. The inf-sup value is approximated using gradually refined triangular meshes. In this first example, the interface is located at the center of the square.

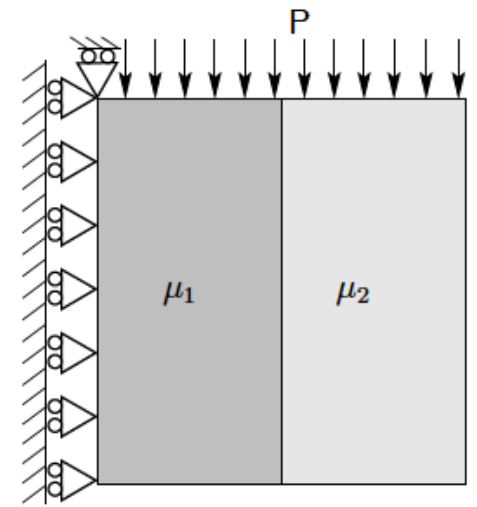

Fig. 17. Problem for the numerical evaluation of the inf-sup.

The sequence of meshes considered here are designed so that the interface passes through the elements (impair number of elements per face). Each face of the square is discretized from 1 to 33 elements (2178 triangular elements on the square). The results are presented in Figure 18 for several approximation spaces. As expected, the Mini element $\left(P_{1}^{+} P_{1}\right)$ for $\mu_{1}=\mu_{2}$ (one material) passes the numerical inf-sup evaluation, as the lower eigenvalue of the system (18) remains bounded away from zero. On the contrary, the $P_{1} P_{1}$ element (which is not LBB compliant) fails this test. The $P_{1}^{+} \oplus P_{1}^{+} * R, P_{1} \oplus P_{1} * H$ and $P_{1}^{+} \oplus P_{1}^{+} * R, P_{1} \oplus P_{1} * R$ spaces seem to pass the numerical inf-sup test. However, the kind of mesh considered here does not exhibit the case of figure presented in Fig. 10. This is why another example is considered in Figure 19. It represents a square composed of two different materials subjected to a constant pressure on its top face. The interface between the two materials is no 


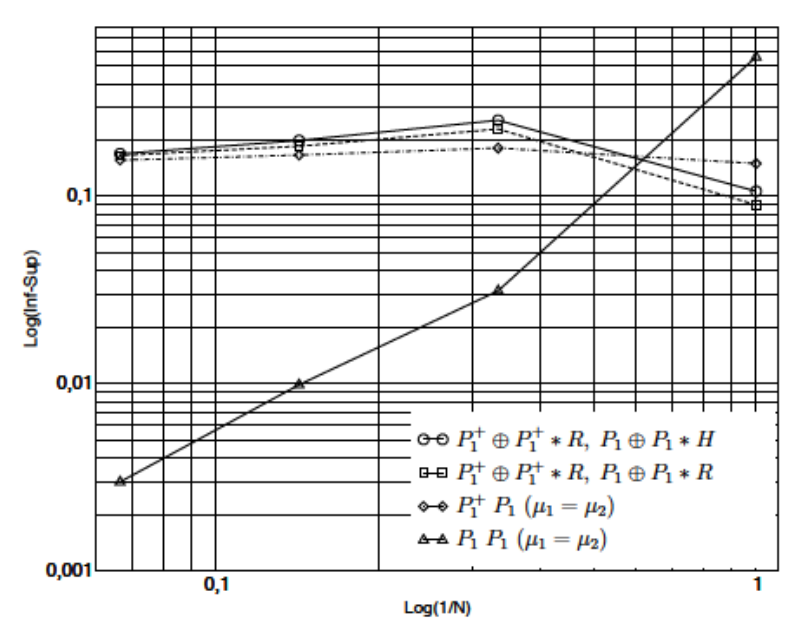

Fig. 18. Evaluation of the inf-sup.

longer at the center of the left side. Its position is parametrized by the parameter $\delta$ (see Fig. 19). First, $\delta$ is set to zero, and the inf-sup is evaluated. As seen

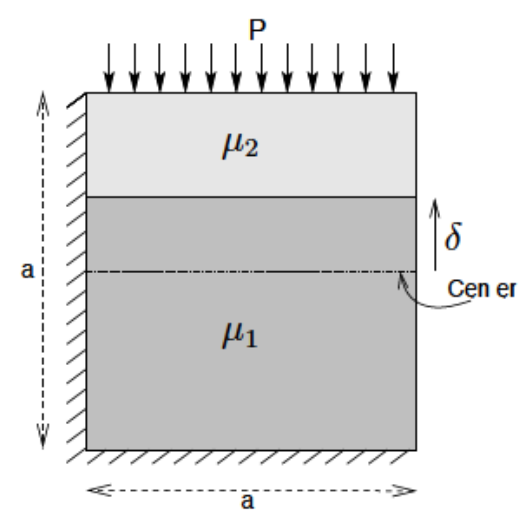

Fig. 19. Second problem for the numerical evaluation of the inf-sup.

in Figure 20, the results are comparable to those obtained in Fig. 18. Then, $\delta$ is set to 0.1 and the mesh is refined to check whether the inf-sup condition is fulfilled. As the interface is unsymmetrical, the phenomenon depicted Fig. 10 will occur at some steps of the discretization. The influence of the position of the interface is clearly shown in figure 21. For Ridge-based enrichment (formulations 3 and 7), no degradation on the inf-sup is observed, the value is stable during refinement. Concerning the Heaviside-based enrichment (formulations 5 and 9), the value of the inf-sup degrades strongly when the element's edges approach the discontinuity (for $1 / N \rightarrow 0.1$ or $1 / N \rightarrow 0.033333$ ). This means that those elements do not pass the inf-sup condition ${ }^{1}$, as the numerical infsup test fails for this situation. Similar results are obtained where only the linear part of the approximation space is enriched (see Figure 22).

1 It is not clear whether the inf-sup parameter does indeed tend to zero as the mesh is refined, but the unstable behaviour of this parameter as well as the non-monotonic convergence leads us to avoid the Heaviside enrichment. 


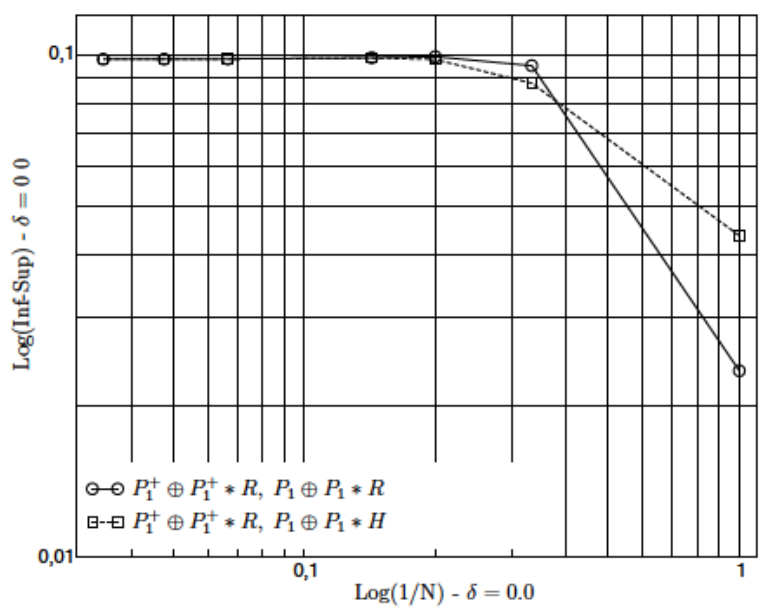

Fig. 20. Evaluation of the inf-sup, problem $2(\delta=0.0)$.

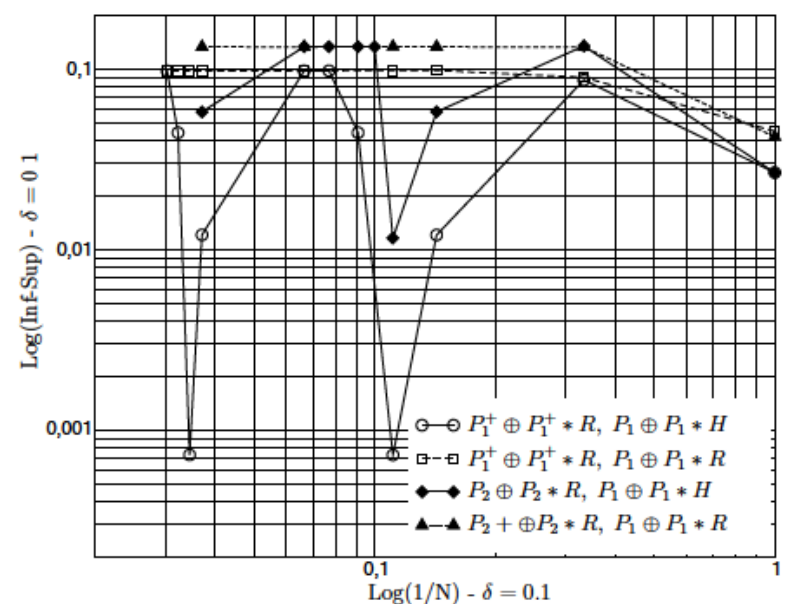

Fig. 21. Evaluation of the inf-sup, problem $2(\delta=0.1)$.

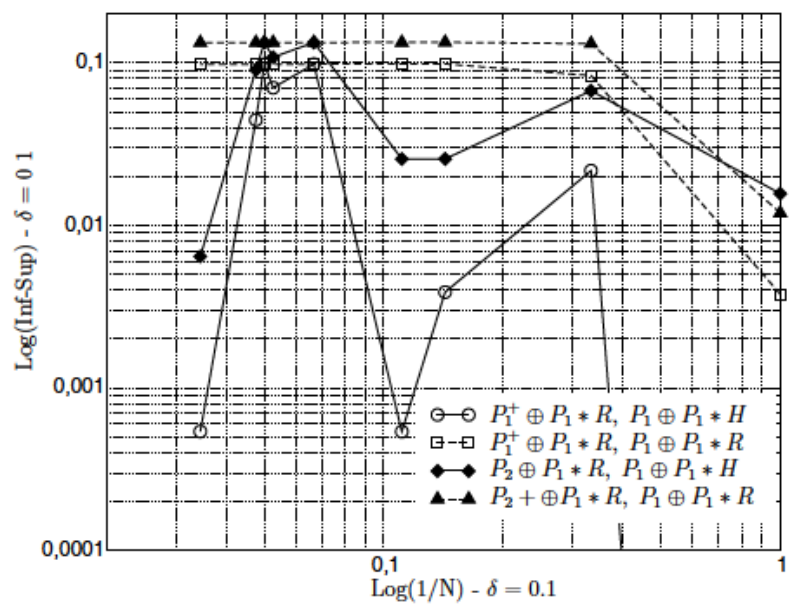

Fig. 22. Evaluation of the inf-sup (linear part enriched), problem $2(\mathrm{R}=0.1)$.

\subsubsection{Conclusion for material interfaces}

The results presented in this section are summarized in Table 1. Strategies involving the enrichment of both displacement and pressure with the ridge 
function are the most effective since they pass the inf-sup test. On the contrary, Heaviside based strategies do not pass this test and should not be considered. The formulations where only the linear part of the displacement is enriched $\left(\mathrm{N}^{\circ} 4\right.$ and 8$)$ with the ridge are interesting, since less degrees of freedom are involved in the approximation. This should be important in the context of an extension to three dimensional studies.

\subsection{Incompressible fracture mechanics}

The resolution of compressible fracture mechanics problems has been extensively studied in the context of the X-FEM for both 2D [39,18,40,32,24] and $3 \mathrm{D}$ fracture mechanics $[21,22]$. The most common enrichment strategy consists in using the asymptotic displacement field as an enrichment for the displacement finite element approximation. In the context of incompressible media, the analytical asymptotic displacement field (Westergaard solution) is shown to be identical to the limit of the compressible one. The asymptotic evolution of the pressure field can be obtained also using the Westergaard solution.

$$
\begin{gathered}
p(r, \theta)=\frac{2 K_{I}}{3 \sqrt{2 \pi r}} \cos \left(\frac{\theta}{2}\right)+\frac{2 K_{I I}}{3 \sqrt{2 \pi r}} \sin \left(\frac{\theta}{2}\right) \\
\phi^{u}=\left\{\sqrt{r} \sin \left(\frac{\theta}{2}\right), \sqrt{r} \cos \left(\frac{\theta}{2}\right), \sqrt{r} \sin \left(\frac{\theta}{2}\right) \sin (\theta),\right. \\
\left.\sqrt{r} \cos \left(\frac{\theta}{2}\right) \sin (\theta)\right\}
\end{gathered}
$$

We use these expressions as an enrichment for the pressure field in the near-tip region. Thus, the enrichment basis for the pressure is expressed as:

$$
\left\{\begin{array}{l}
\phi_{I}^{p}=\frac{1}{\sqrt{r}} \cos \left(\frac{\theta}{2}\right) \\
\phi_{I I}^{p}=\frac{1}{\sqrt{r}} \sin \left(\frac{\theta}{2}\right)
\end{array}\right.
$$

Note that a classical Heaviside enrichment is considered for both pressure and displacement for nodes whose support is fully cut by the crack, and that only the Mini element is considered hereunder.

\subsubsection{Convergence study}

Consider a domain $\Omega=[-1,1] \times[-1,1]$ under tension (see Figure 23). The tensions applied on the boundary of the domain are related to the exact ten- 
sions in an infinite cracked body under mixed mode (using the Westesgaard solution with $\left.K_{I}=1.0, K_{I I}=1.0\right)$. A sequence of gradually refined meshes is considered. Those meshes are built so that the crack passes always through the elements. Two types of meshes are considered: the first one is presented in Figure 23 where the crack tip is located on an element's edge, and the second one (see Figure 23) where it finishes inside an element. Two enrichment strategies are also considered for the near-tip region: first, the topological enrichment where only the nodes whose support contains the crack are enriched, and the so-called 'geometrical' enrichment introduced by Bechet et al. [41] where all the nodes which lie in a circle (of radius 0.1 here) are enriched with crack tip fields. The authors have shown that the convergence rate was improving from $O\left(h^{1 / 2}\right)$ to $O(h)$ with the use of this enrichment (for a given benchmark). The
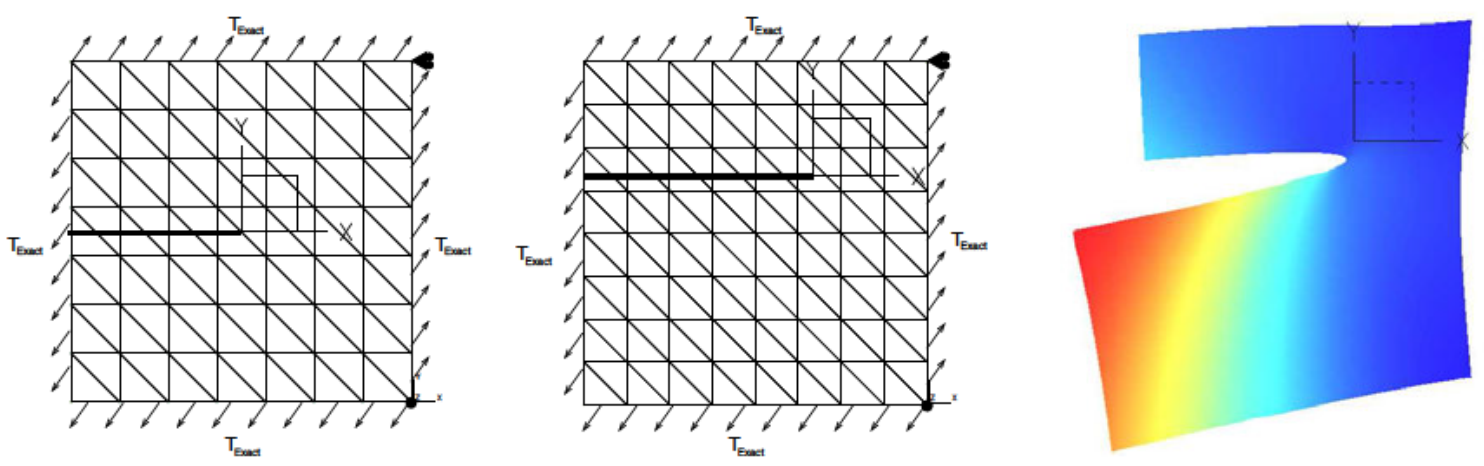

Fig. 23. Domain of interest, mesh $\mathrm{N}^{\circ} 1$ (left), mesh $\mathrm{N}^{\circ} 2$ (center), deformed shape (right).

convergence study are presented in Figure 24 for the first mesh, and Figure 25 for the second. As seen in Figure 24, the convergence rate for both displacement and pressure are in $O\left(h^{1 / 2}\right)$ for the topological enrichment, as expected. The influence of the enrichment is clearly drawn, as it shifts downward the displacement and pressure error curves. For a geometrical enrichment, we obtain results similar to [41], i.e. an improvement of the convergence rate up to $O(h)$ for both pressure and displacement. In this particular case, we obtain a slightly better convergence rate for the pressure. The conclusions are similar for the second mesh.

Note that the case where only the displacement field is enriched has been considered and has shown that the influence of the pressure enrichment is small when dealing with topological enrichment (changes of the initial value of the curve), whereas it is huge when dealing with the geometrical enrichment (degradation of both initial value and convergence rate). This is why, the geometrical enrichment of both pressure and displacement field is the more effective way to model incompressible fracture mechanics. Finally, the case where the enrichment is applied only on the linear part of the approximation is considered in Figure 26. The rate of convergence is shown to be preserved, while degrees of freedom are saved. 


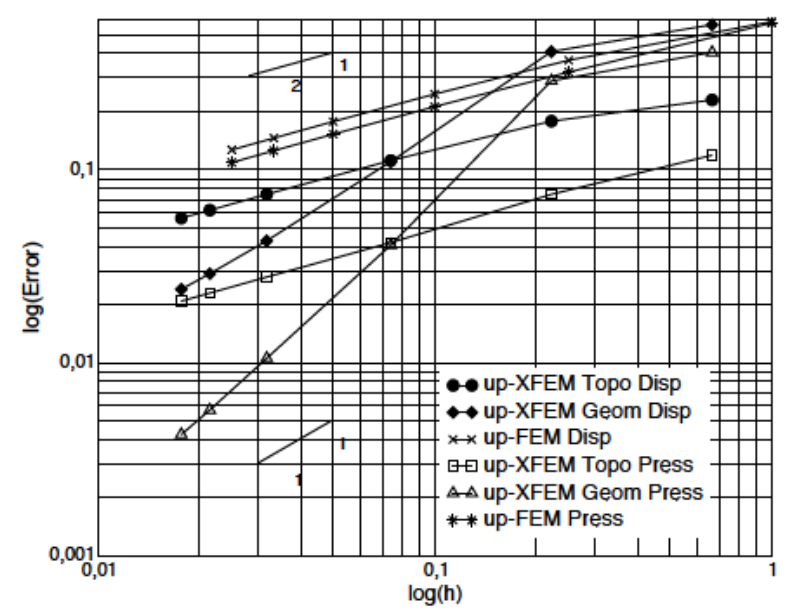

Fig. 24. Convergence rate, structured mesh $\mathrm{N}^{\circ} 1$.

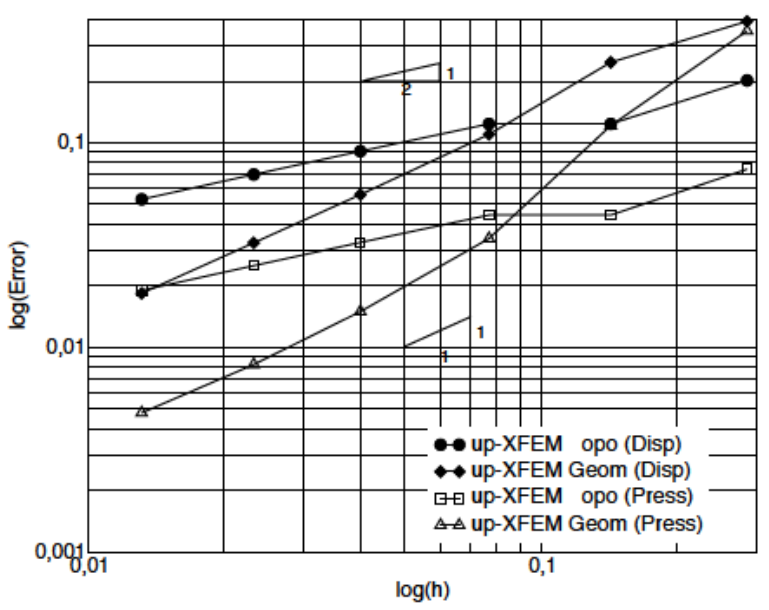

Fig. 25. Convergence rate, structured mesh $\mathrm{N}^{\circ} 2$.

\subsubsection{Numerical Inf-sup test}

The evaluation of the inf-sup condition is performed by considering a single edge notched plate of side length 2.0. A pressure is applied on the upper face, bottom and left faces are held fixed. This loading case is much more drastic for the inf-sup test than the one depicted in Fig. 23. The crack tip is located at $(0.1,0.05)$, so that its relative position to the mesh will change during refinement (see Figure 27). The evolution of the smallest eigenvalue of the inf-sup problem is plotted in Figure 28 for 5 cases of figure: topological enrichment, geometrical enrichment (radius 0.4), classical FEM, topological enrichment (linear part enriched), geometrical enrichment (linear part enriched). The evolution of the eigenvalue tends to a finite value for all curves, showing that the inf-sup condition seems to be fulfilled for both of them. 


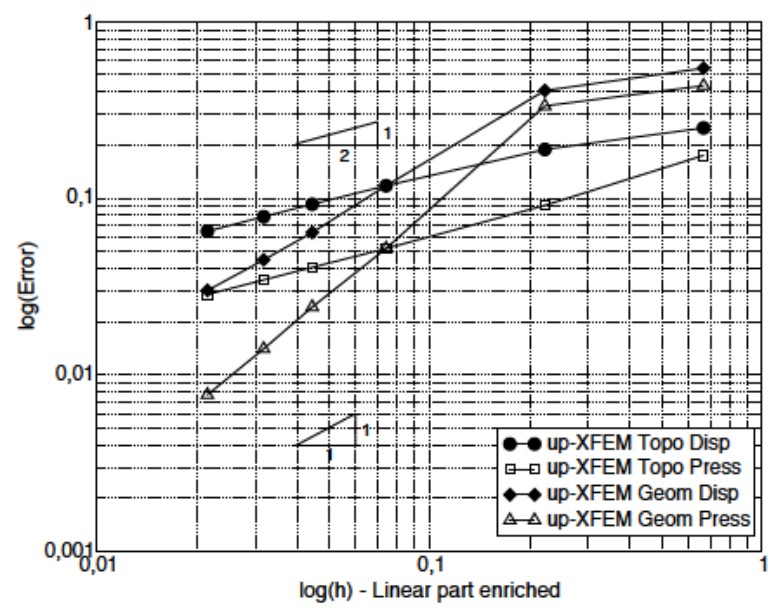

(a)

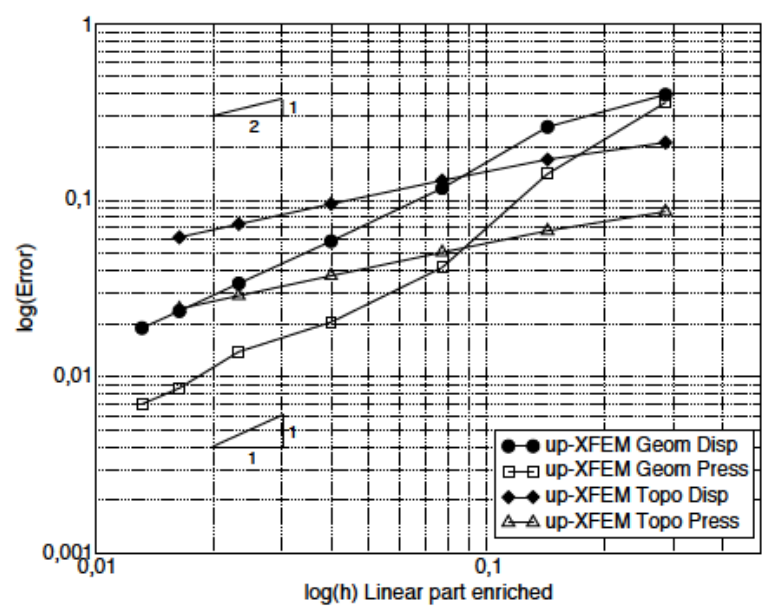

(b)

Fig. 26. Convergence rate, linear part enriched: (a) mesh $\mathrm{N}^{\circ} 1$, (b) mesh $\mathrm{N}^{\circ} 2$.

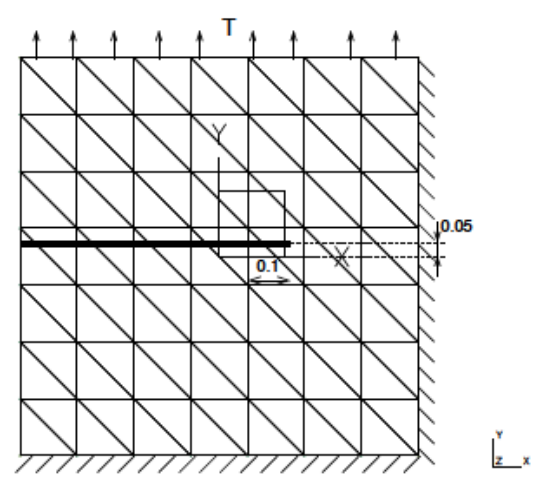

Fig. 27. Fracture inf-sup problem.

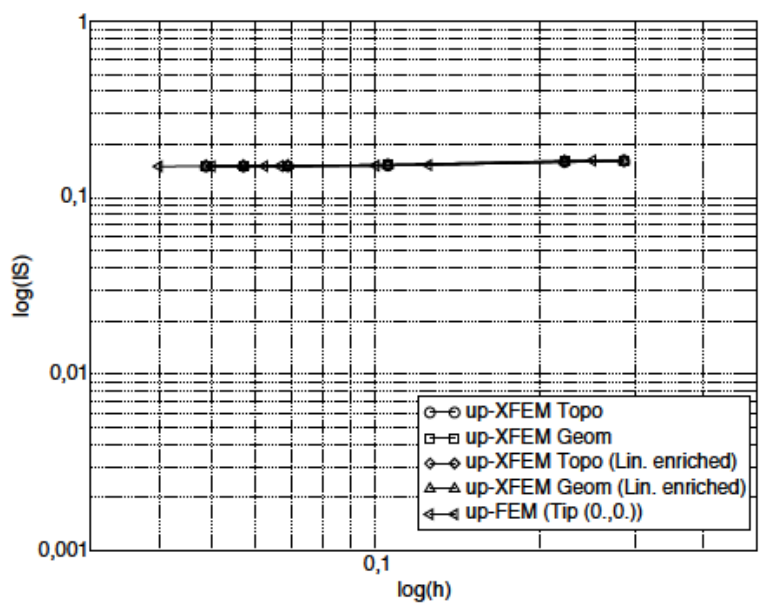

Fig. 28. Fracture inf-sup results.

\subsubsection{Conclusion for fracture mechanics}

As a conclusion, we have seen that the enrichment of both displacement and pressure fields with their asymptotic expressions leads to a stable mixed formu- 
lation. Moreover, the use of the geometrical enrichment leads to an improved convergence case, similar to the compressible rate. Finally, degrees of freedom can be saved by only enriching the linear part of the approximation.

\section{Conclusion}

Some strategies for enriching existing mixed finite element methods have been presented. These strategies are natural extensions of the displacement-based $\mathrm{X}-\mathrm{FEM}$, and are shown to preserve the classical finite element convergence rate. The stability of these strategies has been shown through the numerical inf-sup test. However, quadratic-based elements could not be tested completely, as in some cases the linear interpolation of the level-set leads to a degraded rate of convergence. The construction and the validation of isoparametric quadratic elements will be the subject of a forthcoming paper. The method should also be applied to finite strain mechanics, as the fulfilment of the inf-sup condition seems to be a prerequisite to build efficient large strain formulations [42]. 


\section{References}

[1] D.J. Naylor. Stress in nearly incompressible materials for finite elements with application to the calculation of excess pore pressure. international journal for numerical methods in engineering, 8:443-460, 1974.

[2] T.J.R. Hughes, R.L. Taylor, and J.F. Levy. High Reynolds number, steady, incompressible flows by a finite element method. John Wiley \& Sons, 1978.

[3] S.F. Pawsey and R.W. Clough. Improved numerical integration of thick slab finite elements. International journal for numerical methods in engineering, $3: 275-290,1971$.

[4] TJR Hughes. Generalization of selective reduced integration procedures to anisotropic and nonlinear media. International Journal for Numerical Methods in Engineering, 15:1413-1418, 1980.

[5] RL. Taylor, PJ. Beresford, and EL. Wilson. A nonconforming element for stress analysis. International Journal for Numerical Methods in Engineering, 10(6):1211-1219, 1976.

[6] EL. Wilson, RL. Taylor, WP. Doherty, and J. Ghaboussi. Incompatible displacement models In Numerical and Computer Models in Structural Mechanics. 1973.

[7] JC. Simo and MS. Rifai. A class of mixed assumed strain methods and the method of incompatible modes. International Journal for Numerical Methods in Engineering, 29:1595-1638, 1990.

[8] JC. Simo and F. Armero. Geometrically non-linear enhanced strain mixed methods and the method of incompatible modes. International Journal for Numerical Methods in Engineering, 33:1413-1449, 1992.

[9] B.D. Reddy and J.C. Simo. stability and convergence of a class of enhanced strain methods. SIAM J. Numer. Anal., 32(6):1705-1728, 1994.

[10] F. Brezzi and M. Fortin. Mixed and Hybrid Finite Element Methods. Springer, New York, 1991.

[11] D. Chapelle and K.J. Bathe. The inf-sup test. Computers 83 structures, 47(45):537-545, 1993.

[12] K.J. Bathe. The inf-sup condition and its evaluation for mixed finite element methods. Computers \& Structures, 79:243-252, 2001.

[13] A. Huerta and S. Fernández-Méndez. Locking in the incompressible limit for the element free galerkin method. international journal for numerical methods in engineering, 50, 2001.

[14] J. Dolbow and T. Belytschko. Volumetric locking in the element free galerkin method. Int. J. Numer. Methods Eng., 46(6):925-942, 1999. 
[15] A. Huerta, Y. Vidal, and P. Villon. Pseudo-divergence-free element free galerkin method for incompressible fluid flow. Computer Methods in Applied Mechanics and Engineering, 193(12-14):1119-1136, 2004.

[16] A. Huerta and S. Fernández-Méndez. Enrichment and coupling of the finite element and meshless methods. International Journal for Numerical Methods in Engineering, 48(11):1615-1636, 2000.

[17] J.M. Melenk and I. Babuška. The partition of unity finite element method : Basic theory and applications. Comput. Methods Appl. Mech. Engrg., 139:289$314,1996$.

[18] N. Moës, J. Dolbow, and T. Belytschko. A finite element method for crack growth without remeshing. International Journal for Numerical Methods in Engineering, 46:131-150, 1999.

[19] N. Sukumar, Z.Y. Huang, J.H. Prévost, and Z. Suo. Partition of unity enrichment for bimaterial interfacial cracks. International Journal for Numerical Methods in Engineering, 59:1075-1102, 2004.

[20] E. Budyn, G. Zi, N. Moës, and T. Belytschko. A model for multiple crack growth in brittle materials without remeshing. International journal for numerical methods in engineering, 2004.

[21] N. Moës, A. Gravouil, and T. Belytschko. Non-planar 3D crack growth by the extended finite element and level sets. part I: Mechanical model. International Journal for Numerical Methods in Engineering, 53:2549-2568, 2002.

[22] A. Gravouil, N. Moës, and T. Belytschko. Non-planar 3d crack growth by the extended finite element and level sets. part II: level set update. International Journal for Numerical Methods in Engineering, 53:2569-2586, 2002.

[23] N. Sukumar, N. Moës, T. Belytschko, and B. Moran. Extended Finite Element Method for three-dimensional crack modelling. International Journal for Numerical Methods in Engineering, 48(11):1549-1570, 2000.

[24] J. Dolbow, N. Moës, and T. Belytschko. An extended finite element method for modeling crack growth with frictional contact. Comp. Meth. in Applied Mech. and Engrg., 190:6825-6846, 2001.

[25] J. Dolbow, N. Moës, and T. Belytschko. Modeling fracture in Mindlin-Reissner plates with the eXtended finite element method. Int. J. Solids Structures, $37: 7161-7183,2000$.

[26] N. Moës and T. Belytschko. Extended finite element method for cohesive crack growth. Engineering Fracture Mechanics, 69:813-834, 2002.

[27] G.N. Wells and L.J. Sluys. A new method for modelling cohesive cracks using finite elements. International Journal for Numerical Methods in Engineering, 50:2667-2682, 2001.

[28] J. Réthoré, A. Gravouil, and A. Combescure. An energy-conserving scheme for dynamic crack growth using the extended finite element method. International Journal for Numerical Methods in Engineering, 2005. 
[29] J.E. Dolbow and A. Devan. Enrichment of enhanced assumed strain approximations for representing strong discontinuities : Addressing volumetric incompressibility and the discontinuous patch test. International journal for numerical methods in engineering, 59:47-67, 2004.

[30] G. Legrain, N. Moës, and E. Verron. Stress analysis around crack tips in finite strain problems using the extended finite element method. International Journal for Numerical Methods in Engineering, 63(2):290-314, 2004.

[31] Pedro M. A. Areias and Ted Belytschko. Non-linear analysis of shells with arbitrary evolving cracks using xfem. International Journal for Numerical Methods in Engineering, 62(3):384-415, 2005.

[32] C. Daux, N. Moës, J. Dolbow, N. Sukumar, and T. Belytschko. Arbitrary branched and intersecting cracks with the eXtended Finite Element Method. International Journal for Numerical Methods in Engineering, 48:1741-1760, 2000 .

[33] N. Sukumar, D. L. Chopp, N. Moës, and T. Belytschko. Modeling holes and inclusions by level sets in the extended finite element method. Comp. Meth. in Applied Mech. and Engrg., 190:6183-6200, 2001.

[34] N. Moës, M. Cloirec, P. Cartraud, and J.-F. Remacle. A computational approach to handle complex microstructure geometries. Comp. Meth. in Applied Mech. and Engrg., 192:3163-3177, 2003.

[35] J. Chessa and T. Belytschko. An extended finite element method for two-phase fluids. Journal of Applied Mechanics (ASME), 70:10-17, 2003.

[36] G.J. Wagner, N. Moës, W.K. Liu, and T. Belytschko. The extended finite element method for Stokes flow past rigid cylinders. International Journal for Numerical Methods in Engineering, 51:293-313, 2001.

[37] D. S. Malkus. Eigenproblems associated with the discrete LBB condition for incompressible finite elements. Int. J. Eng. Sci., 19(10):1299-1310, 1981.

[38] D.N. Arnold, F. Brezzi, and M. Fortin. Stable finite element for stokes equations. Calcolo, 21:337-344, 1984.

[39] T. Belytschko and T. Black. Elastic crack growth in finite elements with minimal remeshing. International Journal for Numerical Methods in Engineering, 45(5):601-620, 1999.

[40] D.L. Chopp and N. Sukumar. Fatigue crack propagation of multiple coplanar cracks with the coupled extended finite element/fast marching method. International journal of engineering science, 41:845-869, 2003.

[41] E. Bechet, H. Minnebo, N. Moës, and B. Burgardt. Improved implementation and robustness study of the $\mathrm{x}$-fem method for stress analysis around cracks. International Journal for Numerical Methods in Engineering, Accepted, 2005.

[42] D. Pantuso and K.J. Bathe. On the stability of mixed finite elements in large strain analysis of incompressible solids. Finite elements in analysis and design, 28:83-104, 1997. 


\section{A Analytical solution for curved interface}

We did construct a specific analytical solution to investigate the convergence of the X-FEM. It represents two perfectly bounded rings made from different materials (see figure 7). The loading is chosen such that:

$$
\begin{aligned}
b_{1 r}(r) & =3 W_{3} c^{2}+2 W_{2} c+W_{1} \\
b_{2 r}(r) & =3 V_{3} c^{2}+2 V_{2} c+V_{1} \\
T_{r} & =W_{3} c^{3}+W_{2} c^{2}+W 1 c+W_{0} \\
T_{\theta} & =-2 \frac{\mu_{1} \mu_{2} a^{2} b^{2}}{c}\left(-a^{2} \mu_{1} c^{2}+a^{2} \mu_{2} c^{2}-\mu_{2} b^{2} c^{2}+a^{2} b^{2} \mu_{1}\right)
\end{aligned}
$$

Under these boundary conditions, the stress field components are:

$$
\begin{aligned}
& \sigma_{r r}^{1}=-\left(W_{3} r^{3}+W_{2} r^{2}+W_{1} r+W_{0}\right) \\
& \sigma_{\theta \theta}^{1}=\sigma_{r r}^{1} \\
& \sigma_{r \theta}^{1}=\frac{-2 \mu_{1} \mu_{2} c a^{2} b^{2}}{\left(-a^{2} \mu_{1} c^{2}+a^{2} \mu_{2} c^{2}-\mu_{2} b^{2} c^{2}+a^{2} b^{2} \mu_{1}\right) r^{2}} \\
& \sigma_{r r}^{2}=-\left(V_{3} r^{3}+V_{2} r^{2}+V_{1} r+V_{0}\right) \\
& \sigma_{\theta \theta}^{2}=\sigma_{r r}^{2} \\
& \sigma_{r \theta}^{2}=\frac{-2 \mu_{1} \mu_{2} c a^{2} b^{2}}{\left(-a^{2} \mu_{1} c^{2}+a^{2} \mu_{2} c^{2}-\mu_{2} b^{2} c^{2}+a^{2} b^{2} \mu_{1}\right) r^{2}}
\end{aligned}
$$

The displacement field components are:

$$
\begin{aligned}
& u_{\theta}^{1}(r)=-\frac{c b^{2} \mu_{2}\left(r^{2}-a^{2}\right)}{\left(-a^{2} \mu_{1} c^{2}+a^{2} \mu_{2} c^{2}-\mu_{2} b^{2} c^{2}+a^{2} b^{2} \mu_{1}\right) r} \\
& u_{r}^{1}(r)=0 \\
& u_{\theta}^{2}(r)=-c \frac{-r^{2} a^{2} \mu_{2}+r^{2} a^{2} \mu_{1}+r^{2} \mu_{2} b^{2}-a^{2} b^{2} \mu_{1}}{\left(-a^{2} \mu_{1} c^{2}+a^{2} \mu_{2} c^{2}-\mu_{2} b^{2} c^{2}+a^{2} b^{2} \mu_{1}\right) r} \\
& u_{r}^{2}(r)=0
\end{aligned}
$$

And the pressure evolution is:

$$
\begin{aligned}
& p^{1}(r)=W_{3} r^{3}+W_{2} r^{2}+W_{1} r+W_{0} \\
& p^{2}(r)=V_{3} r^{3}+V_{2} r^{2}+V_{1} r+V_{0}
\end{aligned}
$$


Moreover, in the example section 4.2.1, we have considered:

$$
\begin{aligned}
\mu_{1} & =1 / 3 \quad \mu_{2}=10 / 3 \\
a & =0.4 \quad b=1.0 \quad c=2.0 \\
W_{3} & =-10.0 \quad W_{2}=20.0 \quad W_{1}=-5.0 \quad W_{0}=-10.0 \\
V_{3} & =0.0 \quad V_{2}=-10.0 \quad V_{1}=5.0 \quad V_{0}=0.0
\end{aligned}
$$

\section{B Analytical solution for a straight interface}

The problem (see figure 15) is built so that the interface between the two materials is straight. The loading is chosen such that:

$$
\begin{aligned}
& b_{1 x}=6 \mu_{1} x \\
& b_{1 y}=-6 \mu_{1} y-2 \mu_{2}+3 y^{2} \\
& b_{2 x}=-24 \mu_{2} x \\
& b_{2 y}=24 \mu_{2} y-2 \mu_{2}+6 y^{2} \\
& T_{1 x}=6 \mu_{1} x \\
& T_{1 y}=-6 \mu_{1} y-2 \mu_{2}+3 y^{2} \\
& T_{2 x}=-24 \mu_{2} x \\
& T_{2 y}=24 \mu_{2} y-2 \mu_{2}+6 y^{2}
\end{aligned}
$$

Under these boundary conditions, the stress field components are:

$$
\begin{aligned}
& \underline{\underline{\sigma}}_{1}=\left(\begin{array}{cc}
2 \mu_{1}\left(-3 y^{2}-\frac{2 \mu_{2} y}{\mu_{1}}\right)-y^{3} & \mu_{1}\left(-\left(6 y+\frac{2 \mu_{2}}{\mu_{1}}\right) x+2\right) \\
\mu_{1}\left(-\left(6 y+2 \mu_{2} / \mu_{1}\right) x+2\right) & 2 \mu_{1}\left(3 y^{2}+\frac{2 \mu_{2} y}{\mu_{1}}\right)-y^{3}
\end{array}\right) \\
& \underline{\underline{\sigma}}_{2}=\left(\begin{array}{cc}
2 \mu_{2}\left(12 y^{2}-2 y\right)-2 y^{3} & \mu_{2}\left(-(-24 y+2) x+2 \frac{\mu_{1}}{\mu_{2}}\right) \\
\mu_{2}\left(-(-24 y+2) x+2 \frac{\mu_{1}}{\mu_{2}}\right) & 2 \mu_{2}\left(-12 y^{2}+2 y\right)-2 y^{3}
\end{array}\right)
\end{aligned}
$$

The displacement field is: 


$$
\begin{aligned}
& u_{1 x}=-\left(3 y^{2}+2 \frac{\mu_{2}}{\mu_{1}} y\right) x+2 y-1 \\
& u_{1 y}=y^{3}+\frac{\mu_{2}}{\mu_{1}} y^{2}-1 \\
& u_{2 x}=\left(12 y^{2}-2 y\right) x+2 \frac{\mu_{1}}{\mu_{2}} y-1 \\
& u_{2 y}=-4 y^{3}+y^{2}-1
\end{aligned}
$$

And the pressure evolution is:

$$
\begin{aligned}
& p_{1}=y^{3} \\
& p_{2}=2 y^{3}
\end{aligned}
$$
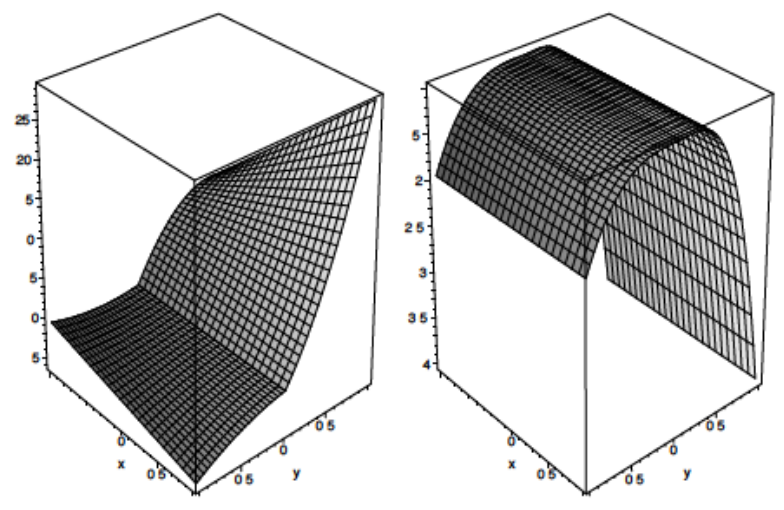

Fig. B.1. Bimaterial problem with straight interface: displacement (left: $u_{x}$, right: $\left.u_{y}\right)$.

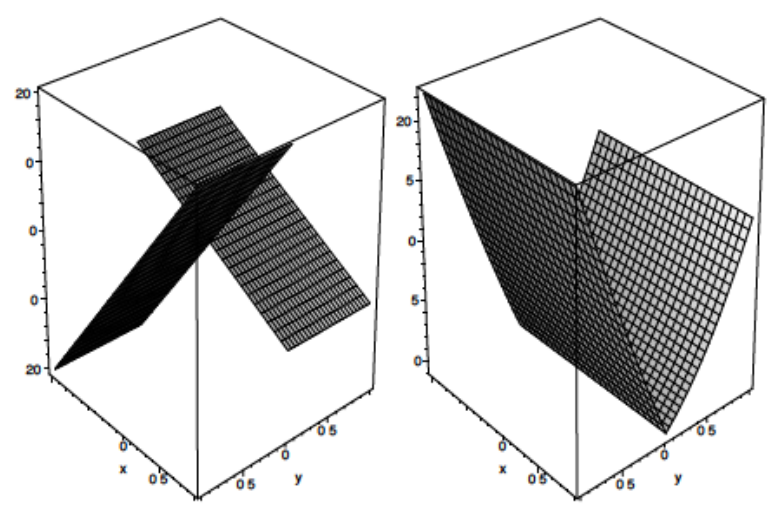

Fig. B.2. Bimaterial problem with straight interface: body forces (left: $b_{x}$, right: $b_{y}$ ). 


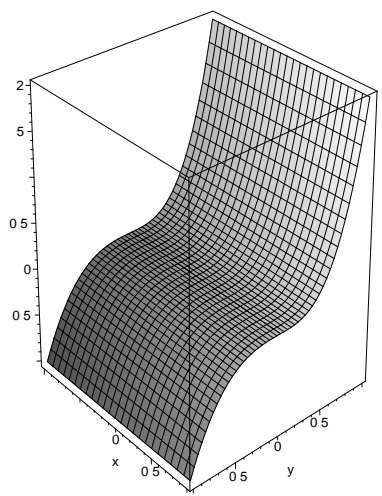

Fig. B.3. Bimaterial problem with straight interface: pressure field. 